Marquette University

e-Publications@Marquette

\title{
Acculturation, Cultural Values, and Latino Parental Beliefs About the Etiology of ADHD
}

Kathryn E. Lawton

Marquette University

\section{Recommended Citation}

Lawton, Kathryn E., "Acculturation, Cultural Values, and Latino Parental Beliefs About the Etiology of ADHD" (2011). Master's Theses (2009-). Paper 118.

http://epublications.marquette.edu/theses_open/118 


\title{
ACCULTURATION, CULTURAL VALUES, AND LATINO PARENTAL BELIEFS ABOUT THE ETIOLOGY OF ADHD
}

\section{by}

Kathryn E. Lawton

A Thesis submitted to the Faculty of the Graduate School, Marquette University, in Partial Fulfillment of the Requirements for the Degree of Master of Science

\author{
Milwaukee, Wisconsin
}

December 2011 


\title{
ABSTRACT \\ ACCULTURATION, CULTURAL VALUES, AND LATINO PARENTAL BELIEFS \\ ABOUT THE ETIOLOGY OF ADHD
}

\author{
Kathryn E. Lawton
}

Marquette University, 2011

\begin{abstract}
Attention-Deficit/Hyperactivity Disorder (ADHD) is one of the most prevalent mental health disorders of childhood, but despite the availability of several evidencebased interventions, Latino children are more likely than non-minority children to have an unmet need for services related to ADHD. Because parental beliefs about the etiology of ADHD likely influence which services are sought, more research is needed to examine this aspect of help-seeking behavior in order to address the unmet need among Latino families. Specifically, research needs to focus on cultural factors that likely influence parental beliefs about the etiology of child behavior problems. Thus, the goal of the current study was to investigate the role of acculturation and cultural values of familismo, respeto, spirituality, and traditional gender roles in explaining parental etiological beliefs about ADHD in Latino parents. Participants in this study included 74 Latino parents. After watching a video portraying a Latino child exhibiting core symptoms and common functional problems associated with ADHD, parents completed a semi-structured interview that assessed etiological beliefs about the behavior of the child in the video, as well as a demographic questionnaire and measures of acculturation and cultural values. Neither Anglo orientation nor Latino orientation was significantly correlated with biopsychosocial or sociological/spiritual etiological beliefs. Additionally, none of the cultural values were significantly correlated with biopsychosocial etiological beliefs. The cultural values familismo and traditional gender roles were significantly positively correlated with sociological/spiritual beliefs. Exploratory analyses also were conducted to further examine sociological/spiritual beliefs. After controlling for SES, familismo and traditional gender roles accounted for $30.5 \%$ of the total variance in sociological/spiritual beliefs about ADHD. Finally, post hoc analyses were conducted to examine individual categories of etiological beliefs. The current study adds to our knowledge about how Latino parents understand child behavior and has important implications for both research and mental health services with Latino parents. The results support the inclusion of etiological beliefs and cultural factors in research examining help-seeking and access to mental health services among Latino families and suggest that the incorporation of alternative etiological beliefs about child behavior may be an important factor in culturally-appropriate mental health services.
\end{abstract}


LIST OF TABLES. .iii CHAPTER

I. INTRODUCTION. 1

A. Help-Seeking Models.............................................

B. Perceptions of the Etiology of ADHD .............................. 3

C. Acculturation and Cultural Values.................................6

D. Current Study ................................................ 10

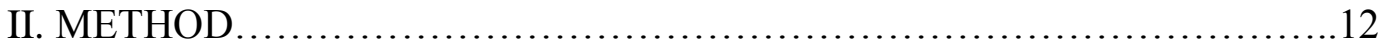

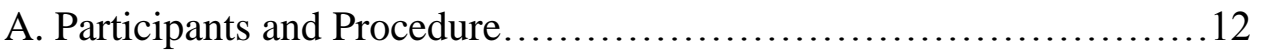

B. Measures..................................................... 13

i. ADHD Behavioral Impairment Video........................13

ii. Belief About Causes Parent Version..........................15

iii. Acculturation Rating Scale of Mexican Americans - II.........16

iv. Mexican American Cultural Values Scale for Adolescents and

Adults................................................... 16

v. Demographic questionnaire................................17

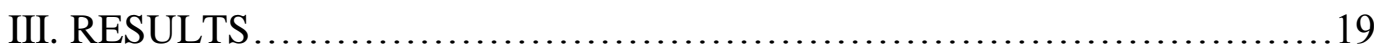

A. Preliminary Analyses........................................... 19

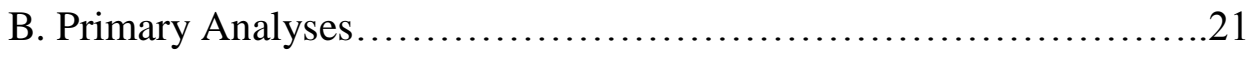

C. Exploratory and Post-Hoc Analyses...............................23

i. Sociological/spiritual beliefs overall factor..................23

ii. Sociological/spiritual individual belief categories.............24

a. Friends............................................... 25 
b. Spiritual......................................27

c. Nature disharmony................................28

IV. DISCUSSION .................................................. 30

A. Acculturation and Etiological Beliefs.............................31

B. Cultural Values and Etiological Beliefs.............................. 32

i. Biopsychosocial beliefs................................32

ii. Sociological/spiritual beliefs..........................32

a. Familismo.......................................33

b. Spirituality......................................34

c. Traditional gender roles...........................34

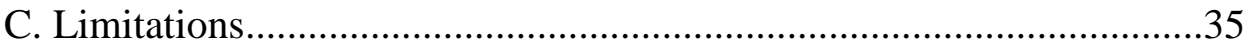

D. Implications and Future Directions...............................................36

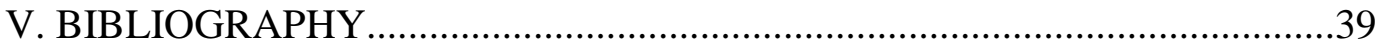




\section{LIST OF TABLES}

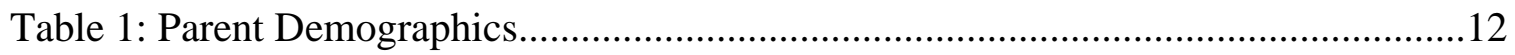

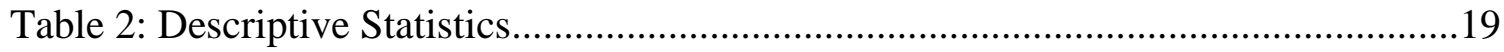

Table 3: Correlations for SES and generational status with ARMSA Subscales, MACVS

Subscales, and Biopsychosocial and Sociological/Spiritual Beliefs..............................21

Table 4: Correlations for ARMSA Subscales and MACVS Subscales with Biopsychosocial and Sociological/Spiritual Beliefs................................................22

Table 5: Summary of Hierarchical Regression Analyses for Sociological/Spiritual Beliefs.

Table 6: Correlations for Independent Variables with Individual Sociological/Spiritual BAC Categories.

Table 7: Summary of Hierarchical Regression Analyses with Individual

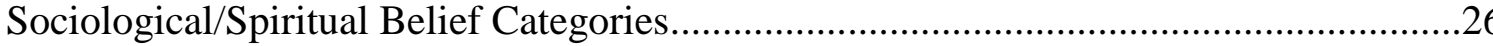




\section{Introduction}

Attention-Deficit/Hyperactivity Disorder (ADHD) is one of the most prevalent mental health disorders of childhood, occurring in 3-7\% of school-age children in the United States (APA, 2000), and is associated with many functional difficulties throughout childhood, adolescence, and adulthood, such as academic underachievement, peer difficulties, family conflict, and lower rates of professional employment (Bagwell, Molina, Pelham \& Hoza, 2001; Biederman \& Faraone, 2006; Johnston \& Mash, 2001; Pelham, Fabiano \& Massetti, 2005). Several evidence-based interventions have been developed to treat ADHD, including both psychosocial and psychopharmacological interventions (Pelham \& Fabiano, 2008). Although these evidence-based treatments are widely available (Pelham \& Fabiano, 2008), there is growing evidence that Latino children have a greater unmet need for mental health services relating to ADHD (Bussing, Zima, Gary \& Garvan, 2003; Zimmerman, 2005) and are assessed and treated at a lower rate than non-minority children (Eiraldi, Mazzuca, Clarke \& Power, 2006; LeFever, Dawson \& Morrow, 1999). Examination of help-seeking models may help explain this discrepancy.

\section{Help-Seeking Models}

Models of help-seeking behavior have been established to address the process through which individuals decide to seek mental health services. The first model, the Behavioral Model of Health Service Use, was originally developed to address factors relating to families' utilization of health services and identified predisposing characteristics, enabling resources, and need as factors that could explain or predict 
service utilization (Anderson, 1995). This model has been continually expanded and revised to explain the help-seeking process for many different groups with different presenting problems, including children and adolescents (Srebnic, Cauce, \& Baydar, 1996) and ethnically-diverse groups (Cauce et al., 2002). Based on this original model, Eiraldi and colleagues (2006) proposed a help-seeking model for service utilization among ethnic minority children with ADHD that divides the process of help-seeking into four stages: problem recognition, decision to seek help, service selection, and service utilization. According to this model, discrepancies between need for services and service utilization among Latino children are the result of both access barriers, as well as individual and cultural factors that may influence the parental decision to seek treatment at any point in the help-seeking process (Eiraldi et al., 2006; Snowden \& Yamada, 2005).

Access barriers are aspects of the mental health system that limit the availability of and access to services, including factors such as socioeconomic status (SES), insurance coverage, and language barriers, all of which may be particularly problematic for Latinos. Families with lower incomes and uninsured families report experiencing more barriers to service utilization and are more likely to have an unmet need for services (Bussing et al., 2003; Kataoka, Zhang, \& Wells, 2002). Additionally, individuals with limited English proficiency may be unable to communicate with service providers and as a result, may be less likely to seek services (Derose \& Baker, 2000). Individual factors, such as demographic variables, also have been examined. For example, research suggests that parents are less likely to seek services for girls or younger children (Bussing et al., 2003). Parental and family factors, such as marital conflict, family size, and parental education 
also may be important factors in the decision to seek services (Srebnic, Cauce, \& Baydar, 1996).

Identifying cultural factors that influence parental decisions to seek services for their child also is essential to understanding discrepancies that exist between the need for services and service utilization among Latinos. Cultural norms for appropriate child behavior may affect the help-seeking process, particularly the problem recognition stage. Before parents choose to seek treatment for their child, they must first recognize their child's behavior as impairing and in need of outside help. Research has demonstrated that expectations for developmentally appropriate behavior vary across ethnic groups (Gidwani, Opitz, \& Perrin, 2006). It is possible that different patterns of service utilization among ethnic groups may be the result of differing expectations for child behavior, as well as differing parental perceptions about the cause of such behavior.

\section{Perceptions of the Etiology of ADHD}

Parental perceptions regarding the etiology of child behavior seem particularly important to consider. Once parents have identified a problem, their perception of the cause of the problem likely influences how they respond and what type of services they seek. Differing parental beliefs about the etiology of ADHD may explain some of the discrepancy between need for services and service utilization among ethnic minorities. Parents who do not believe their child's inattention and hyperactivity/impulsivity are the result of biological or psychological factors may be less likely to seek medical or psychological services and may be more likely to seek services that fit with their explanation for the problem, such as seeking guidance from a spiritual leader (Yeh, McCabe, Hough, Lau, Fakhry, \& Garland, 2005). Research has shown that parents who 
believe that their child's problems originate from physical causes or trauma are more likely to seek mental health services, whereas parents who believe that the problems resulted from relational issues, family issues, or the child's personality are less likely to seek such services (Yeh et al., 2005).

The current scientific conceptualization of the etiology of ADHD emphasizes the role of neurological and genetic factors in causing ADHD (for a review, see BrassettHarknett \& Butler, 2007). Although psychosocial factors contribute to aspects of the disorder such as its severity, continuity, outcome, and comorbidities, there is little evidence that these factors contribute to the etiology of ADHD, as was once suspected (Barkley, 2006). Despite the strong evidence supporting genetic and neurological underpinnings of ADHD in the psychological literature, the public is relatively uninformed about ADHD and its etiology. McLeod, Fettes, Jensen, Pescosolido, and Martin (2007) found that approximately one-third of participants had never heard of ADHD, and fewer than half were able to provide additional information about the disorder. Ethnic minorities in this study were even less likely to have specific knowledge about ADHD. This study is consistent with research that has found that the public is not well-informed about mental health disorders in general (Jorm, 2000), and that ethnic minorities tend to be even less informed (Bussing, Shoenberg, \& Perwien, 1998a).

While a general lack of knowledge about ADHD may influence parental understanding of the etiology of inattentive and hyperactive/impulsive behavior in their child, other research suggests that there may be cultural factors that influence these beliefs. Yeh, Hough, McCabe, Lau, and Garland (2004) examined three broad categories of beliefs about the causes of child problems. Parents of children receiving services for 
various emotional and behavioral problems were asked about several etiological explanations for their child's behavior problems, including biological, psychological, and social causes, as well as spiritual and nature disharmony causes (Yeh et al., 2004).

Results indicated that ethnic minority parents were less likely than White parents to believe their child's problems were caused by biological, psychological, or social factors. Personality factors were cited most often by Latino, African American, and White parents as contributing to their child's behavior. In addition, Latino parents were more likely than other groups to identify family issues as a factor in their child's behavior.

Bussing and colleagues examined parental etiological beliefs for ADHD in different ethnic groups (Bussing et al., 1998a; Bussing Gary, Mills, \& Garvan, 2008; Bussing, Schoenberg, Rogers, Zima \& Angus, 1998b). Results indicated that African American parents were more likely to label inattentive and hyperactive/impulsive behavior as "problem" behavior, to consider their child "bad," and to believe that sugar had a role in their child's behavior, whereas White parents were more likely to use a medical label to describe their child's behavior (Bussing et al., 1998a; Bussing et al., 1998b). Additionally, they found that African American parents were less likely to have heard of ADHD, and few parents were aware of special services available to children with ADHD (Bussing et al., 2008).

This preliminary research provides an initial foundation for understanding Latino parental beliefs about the etiology of ADHD; however, several limitations of this research should be noted. Many studies examining ethnic differences did not include Latinos in their samples (Bussing et al., 1998; Bussing et al., 2008). Studies that did include Latinos only included individuals who were proficient in English (Pham, Carlson, 
\& Kosciulek, 2009). Given that a large percentage of Latinos living in the United States speak Spanish as their primary language (Marotta \& Garcia, 2003), findings from these studies may not generalize to a wider Latino population. In addition to limited sampling of Latinos, racial or ethnic status has been used as a proxy for culture. No research to date has examined actual cultural factors that may influence Latino parental beliefs about the etiology of ADHD.

\section{Acculturation and Cultural Values}

Acculturation is defined as the process of cultural learning and change that occurs when two different groups, usually an immigrant group and a cultural majority, come into contact with each other, resulting in changes in both groups (Antshel, 2002; Berry, 2001; Marín \& Gamba, 2003). Current theories highlight the mutual process that occurs in both groups, involving each group's own beliefs and cultural identity, as well as perceptions of and contact with the other group (Berry, 2001). According to this bidimensional framework, immigrants' identification with a new culture is independent of their identification with their original culture (Lara, Gamboa, Kahramanian, Morales, \& Bautista, 2005). Thus, Latino immigrants, for example, who identify strongly with their traditional Latino culture may also identify strongly with American culture, may identify strongly with only one culture, or with neither. Because the process of acculturation may change or influence cultural values, beliefs, and norms (Koneru, Mamani, Flynn, \& Betancourt, 2007), level of acculturation may play a role in parental etiological beliefs about ADHD. Parents who identify strongly with American culture may adopt the beliefs of the dominant culture, whereas parents who identify with Latino culture may hold beliefs consistent with Latino culture (Ramos-Sanchez \& Atkinson, 2009). 
The process of cultural learning that occurs among immigrants has been conceptualized as occurring on three levels; the first two levels include behavioral changes, and the third level includes changes in values and norms (Marín, 1992). Initially, an immigrant may learn about the host culture's history and tradition and may make changes in the type of food and media he/she consumes; these behavioral changes are generally on a superficial level. Next, behaviors associated with the individual's social life may change; the individual may change his or her preference of language and preference for the ethnicity of friends and neighbors. Finally, more significant changes, such as changes in the individual's values and/or the adoption of the values and norms of the host culture, may occur (Marín, 1992). Thus, examining cultural values held by Latino parents is essential to understanding their etiological beliefs about mental illness.

One of the most salient cultural values among Latinos is familismo, often considered to be a hallmark of Latino culture (Schwartz, 2007). Familismo refers to the strong attachment to and reliance on the nuclear and extended family (Marín \& Gamba, 2003). It is characterized by feelings of loyalty, honor, and unity, and includes components such as interdependence, respect toward authority figures, and personal sacrifice for the good of the family (Antshel, 2002; Schwartz, 2007). Familismo has been conceptualized as having three main components: perceived obligation to provide support to extended family, reliance on family for support, and use of family as behavioral and attitudinal referents (Marín \& Marín, 1991). Some research has suggested that Latinos may view health and illness in the context of their family and as part of a complex network (Reichman, 2006). Perceptions of health may be grounded in an individual's relationship with family (Mendelson, 2002). Therefore, Latino parents may not view 
their child's inattention and hyperactivity/impulsivity as something inherent to the child but rather the result of some conflict within the family. Additionally, the extended family is likely to play a role in helping the parents to understand a child's behavior and its cause. If no one in the family or social network recognizes a child's inattentive or hyperactive/impulsive behaviors as being the result of a psychological condition, parents will be less likely to believe the cause of these behaviors is psychological (Bussing et al., 1998b).

Another important value in Latino culture is respeto, which refers to respect in interpersonal relations. In Latino culture, it is expected that authority figures and elders are always treated with respect, and children are expected to be respectful toward their parents and strangers (Marín \& Marín, 1991). Some of the acting out behavior that is often associated with ADHD may be perceived as disrespectful by Latino parents. The cultural emphasis placed on respeto may make these behaviors particularly troublesome to Latino parents (Livingston, 1999) and may influence what they perceive the cause of such behaviors to be. If they perceive that the behaviors are a deliberate attempt to be disrespectful, rather that resulting from a neurological condition, they may be less likely to seek mental health services and more likely to try to manage the behavior within the family.

Additionally, spirituality may influence parental beliefs about the etiology of ADHD. Although it is difficult to determine a precise definition of spirituality, it is often conceptualized as heightened awareness, consciousness, and connectedness with all beings (Cervantes \& Parham, 2005) and with a universal being (Campesino \& Schwartz, 2006), with beliefs existing on a continuum (Baez \& Hernandez, 2001). Spirituality may 
take the form of religious faith or may be expressed through other means (Drench, Noonan, Sharby, \& Ventura, 2007). Although spirituality is integral to Latino culture (Campesino \& Schwartz, 2006), many Latinos report that religiosity is not central to their spirituality (Mendelson, 2002; Musgrave, Allen, \& Allen, 2002). Latino culture's emphasis on spirituality often leads Latinos to view health holistically, as a balance between mind, body, and spirit; health and illness are often thought to come from God (Musgrave, Allen, \& Allen, 2002). Spirituality is often an integral part of their definitions of health and illness (Mendelson, 2002). Therefore, spirituality and spiritual beliefs may influence parental etiological beliefs about problem behavior in their children. They may view the disruptive behavior as an indication that the child is out of balance and seek advice from a spiritual leader (Yeh et al., 2005), rather than seeking psychological services.

Finally, another important cultural variable to examine is the extent to which Latino parents adhere to traditional gender roles. Traditional gender roles reflect differential expectations for male and females; the word traditional is used to distinguish between previous views of gender roles and the more flexible definitions of gender and gender roles that have arisen since the women's movement in the 1970s (Cuellar \& Paniagua, 2000). Traditional gender roles represent a patriarchal organization of gender. Expectations for men include being the provider for the family, being strong and in control, and being the head of the household; expectations for women include having a caretaking role for children and the elderly in the family (Knight et al., 2009; Marín \& Marín, 1991). Adherence to traditional gender roles may influence how both mothers and fathers understand child behavior and what cause they ascribe to it. Parents may 
have different views about who should be responsible for discipline and different expectations for appropriate behavior based on the gender of their child, and inattentive and hyperactive-impulsive behavior may be understood differently according to these expectations.

\section{Current Study}

Despite the availability of evidence-based treatments for ADHD, there is an unmet need for services relating to ADHD among ethnic minority families (Bussing et al., 2003; Zimmerman, 2005). In order to address this unmet need, research is needed to understand factors that may contribute to this gap between need for services and service utilization, particularly among the growing Latino population. Because parental beliefs about the etiology of ADHD likely influence which services they seek, more research is needed to address this aspect of help-seeking behavior. Specifically, research needs to move beyond just examining differences between ethnic minority groups and focus on more specific cultural factors that likely influence parental beliefs about the etiology of child behavior problems. Thus, the goal of the current study was to investigate the role of culture in explaining parental etiological beliefs about ADHD in Latino parents.

It was hypothesized that acculturation would be associated with parental etiological beliefs of ADHD, such that higher Anglo orientation (i.e., identifying with mainstream American culture) would be associated with endorsement of more biopsychosocial beliefs, whereas higher Latino orientation (i.e., identifying with Latino culture) would be associated with more sociological/spiritual beliefs about the etiology of ADHD. It also was hypothesized that the cultural values of familismo, respeto, spirituality, and traditional gender roles would be related to sociological and spiritual 
etiological beliefs of ADHD. Specifically, it was hypothesized that there would be a positive relationship between these cultural values and sociological/spiritual causes of ADHD and a negative relationship between the cultural values and biopsychosocial causes. Finally, exploratory analyses were planned to determine which cultural factors would be most predictive of parental etiological beliefs of ADHD. 


\section{Method}

\section{Participants and Procedure}

Participants in this study included 74 Latino parents who were recruited as part of a larger study examining cultural influences on ADHD problem recognition. Inclusion criteria were self-identification as Latino and having at least one child between the ages of 5 and 12 years. The majority of participants in this sample were of Mexican origin $(87.8 \%)$, female $(63.5 \%)$ and married $(73 \%)$; the mean age was $37.3(\mathrm{SD}=5.3)$. Most participants have lived in the United States for more than 10 years (71.6\%) and spoke only Spanish (40.5\%) or primarily Spanish and some English (41.9\%). See Table 1 for more demographic information.

Table 1

Parent Demographics

Age, $M(S D)$

$37.03 \quad(5.27)$

Gender, $n(\%)$

Female

$47 \quad(63.5)$

Male

$25 \quad$ (33.8)

Marital Status, $n(\%)$

Married

$54 \quad(73.0)$

Unmarried

18 (27.0)

Education, $n(\%)$

Less than high school or some high school 38

Graduated high school or GED 19

Some college or specialized training 7

College or graduate degree

$6 \quad(8.1)$

Income, $n(\%)$

$\begin{array}{lrc}\text { Less than } \$ 20,000 & 27 & (36.5) \\ \$ 20,001-40,000 & 33 & (44.6) \\ \$ 40,001-60,000 & 6 & (8.2)\end{array}$


$\$ 60,001-80,000$

Country of Origin, $n(\%)$

Mexico

Puerto Rico

Other

Time in US, $n(\%)$

$1-5$ years

6-10 years

More than 10 years

Born in US

Language, $n(\%)$

Only Spanish

Primarily Spanish, some English

Bilingual

Primarily English, some Spanish
2

$65 \quad(87.8)$

$3 \quad(4.1)$

$4 \quad(5.4)$

$1 \quad(1.4)$

$14 \quad(18.9)$

$54 \quad(71.6)$

$4 \quad(5.4)$

$30 \quad(40.5)$

$31 \quad$ (41.9)

$10 \quad(13.5)$

$1 \quad(1.4)$

$N=72$

Participants were recruited from local Catholic churches in a culturally-diverse city in Southeastern Wisconsin. The study took approximately 90 minutes to complete and was conducted at the recruitment locations (i.e., local churches) during church events. Following informed consent procedures, participants watched a 10-minute video portraying a Latino child exhibiting core symptoms and common functional problems associated with ADHD. Following the video, parents completed a semi-structured interview that assessed etiological beliefs about the behavior of the child shown in the video. A demographic questionnaire, as well as measures of acculturation and cultural values, also were administered following the interview. All measures were available in both English and Spanish and were randomized.

\section{Measures}


ADHD Behavioral Impairment Video (BIV). Parents were shown the ADHD BIV, which was approximately 10 minutes in length and portrayed a Latino child exhibiting the core symptoms and common functional problems associated with ADHD. Given that ADHD is generally more frequent in males and typically diagnosed in schoolaged children, the child in the video was a 9-year-old boy. Core symptoms (e.g., having difficulty sustaining attention, being easily distracted, fidgeting with hands or feet, having difficulty waiting his turn) and common functional problems (e.g., family conflict, academic difficulties, peer rejection) were shown in three settings: at home, at school, and with peers. For example, the child was shown not listening to parental instructions to clean up toys, not completing tasks, such as chores and homework, and not getting along with his parents and siblings at home. At school, the child was shown having difficulty sustaining attention and listening to the teacher's instructions, fidgeting and moving around in the classroom, and not completing his school work. Finally, with peers, the child was shown irritating other children by interrupting their game and being rejected by other children. Three clips for each of the three settings were shown.

Prior to beginning the current study, a three-phase pilot study was conducted with the BIV to determine the symptoms and functional problems that were consistently endorsed following viewing of the video. First, the research team who developed the BIV viewed the video and completed a checklist of symptoms and functional problems that they considered to be present. Next, clinical psychology doctoral students specializing in children viewed the BIV and completed the checklist; items endorsed were generally consistent with those endorsed by the research team. Finally, a community sample of ten mothers of school-aged children viewed the BIV and 
completed the checklist. Symptoms and functional problems endorsed by at least $70 \%$ of the mothers were considered present. All of the symptoms and functional problems ultimately considered present also had been endorsed by the research team in the first phase of the pilot, and $85 \%$ of the symptoms and all of the functional problems had been endorsed by the doctoral students in the second phase of the pilot.

Belief About Causes Parent Version (BAC; Yeh \& Hough, 2005). The BAC is a semi-structured interview that was used to measure etiological explanations for ADHD. It assesses eleven categories of etiological beliefs and is available in both English and Spanish. Parents were asked to answer yes or no to broad questions assessing etiological beliefs. Endorsement of a broad question prompted additional questions from within that category, with the exception of the Prejudice category. The categories of etiological beliefs include Physical Causes (i.e., genetics or heredity), Personality (i.e., child's characteristics), Relational Issues (i.e., problems with social skills), Familial Issues (i.e., conflict within the family), Trauma, Friends, American Culture, Prejudice, Economic Problems (i.e., not having enough money for food, clothing, etc.), Spiritual Causes (i.e., bad luck, the will of God), and Nature Disharmony (i.e., disruption of child's energy or vital flow). Confirmatory factor analysis has revealed a two factor structure of the measure, which will be used as the primary dependent variables in the proposed study. Physical Causes, Personality, Relational Issues, Familial Issues, and Trauma fall into the biopsychosocial category. Friends, American Culture, Prejudice, Economic Problems, Spiritual Causes and Nature Disharmony fall into the sociological/spiritual category (Yeh et al., 2005). Construct validity of the measure is supported by the different patterns of 
endorsement among several racial and ethnic groups (Yeh et al., 2004; Yeh et al., 2005). The measure displayed good reliability in the current study (Cronbach's alpha $=.92$ ).

Acculturation Rating Scale of Mexican Americans-II (ARSMA-II; Cuellar, Arnold, \& Maldonado, 1995). The ARSMA-II is a two dimensional measure of acculturation that measures an individual's Mexican orientation (Mexican Orientation Subscale $[\mathrm{MOS}]$ ) and Anglo orientation (Anglo Orientation Subscale [AOS]). The measure consists of 30 items that assess language use and preference, ethnic identity and classification, and ethnic interaction and is available in both English and Spanish. The 30 items are measured on a 5-point Likert scale ranging from 1 (not at all) to 5 (extremely often or almost always). Cuellar, Arnold, and Maldonado (1995) reported split-half reliability of .77 for the AOS and .84 for MOS and coefficient alphas of .83 for the AOS and .88 for the MOS. Concurrent validity was assessed with the original version of the ARSMA, and the two tests obtained a correlation coefficient of .89. Cuellar, Arnold, and Maldonado (1995) also reported good construct validity, demonstrated by the measure's ability to differentiate five generational levels of Mexicans and Mexican Americans. The current study modified the ARSMA-II by substituting "Latino" for "Mexican" or "Mexican American" in an effort to make it more suitable to use with a wider Latino population. This method has been used previously and maintains the psychometric properties of the measure (Steidel \& Contreras, 2003). The measure displayed good reliability in the current study (Cronbach's alpha $=.78)$.

\section{Mexican American Cultural Values Scale for Adolescents and Adults}

(MACVS; Knight et al., 2009). The MACVS is a 50-item measure of several cultural constructs relevant to Latino and mainstream American culture and is available in both 
English and Spanish. The scale measures familism, divided into three subscales of familism support (i.e., "parents should teach their children that the family always comes first"), familism obligations (i.e., "children should be taught that it is their duty to care for their parents when their parents get old"), and familism referent (i.e., "children should always do things to make their parents happy"), as well as respect (i.e., "children should respect adult relatives as if they were parents"), religion (i.e., "God is first, family is second"), and traditional gender roles (i.e., "families need to watch over and protect teenage girls more than teenage boys"). These subscales are combined into an overall Mexican American values scale. The scale also measures mainstream values of material success, independence and self-reliance, and competition and personal achievement that are combined into an overall mainstream values scale. Items are measured on a 5-point Likert scale ranging from 1 (not at all) to 5 (completely). The MACVS has shown good psychometric properties; Cronbach's alphas are .79 for both mothers and fathers for the composite of the familism subscales, .88 for both on the overall Mexican American values scale, and .81 and .82 for mothers and fathers, respectively, on the overall mainstream values scale. The measure also has demonstrated adequate construct validity, as it is related to several similar constructs, such as ethnic pride, ethnic socialization, and country of origin (Knight et al., 2009). The current study used the overall familism subscale and the respect, religion, and traditional gender roles subscales. Despite the name of the measure, specific questions do not specify a particular Latino group (i.e., Mexican), and therefore the measure is likely acceptable to use with a wide Latino population. The measure displayed good reliability in the current study (Cronbach's alpha $=.86)$. 
Demographic questionnaire. A demographics questionnaire was administered to collect general information about each participant, such as age, gender, generational status, and educational attainment. Generational status was measured categorically; participants were considered first generation if they had been born outside the US, second generation if they were born in the US but at least one of their parents had been born outside the US, and third generation if they were born in the US and both of their parents had been born in the US. None of our participants were of a higher generational status. 


\section{Results}

\section{Preliminary Analyses}

Descriptive statistics for each of the variables are presented in Table 2. Families in the current study tended to be of lower socioeconomic status (SES), as indicated by a mean of 27.39 (10.07) on a scale ranging from 8 to 66 , with higher scores indicating higher SES (Hollingshead, 1975). Most parents in the sample were first generation $(M=$ $1.07, S D=.31)$. Parents reported higher orientation toward Latino culture $(M=4.36$ out of $5, S D=.45)$ than toward Anglo culture $(M=2.69, S D=.75)$. Additionally, parents reported moderate to strong adherence to Latino cultural values of familism $(M=4.39$ out of $5, S D=.43)$, traditional gender roles $(M=2.78, S D=.97)$, religion $(M=4.65, S D$ $=.40)$, and respect $(M=4.24, S D=.49)$. Parents endorsed $69.08 \%$ of the items that compose the biopsychosocial etiological beliefs subscale and $32.5 \%$ of the items that compose the sociological/spiritual beliefs subscale.

Table 2

Descriptive Statistics

\begin{tabular}{|c|c|c|}
\hline Generational Status, N (\%) & & \\
\hline First & 68 & $(94.4)$ \\
\hline Second & 3 & $(4.1)$ \\
\hline Third & 1 & $(1.4)$ \\
\hline $\mathrm{SES}^{*}, \mathrm{M}(\mathrm{SD})$ & 27.39 & $(10.07)$ \\
\hline ARSMA AOS, M (SD) & 2.69 & $(0.75)$ \\
\hline ARSMA LOS , M (SD) & 4.36 & $(0.45)$ \\
\hline MACVS Familism, M (SD) & 4.39 & $(0.43)$ \\
\hline MACVS Gender Roles, M (SD) & 2.78 & $(0.97)$ \\
\hline
\end{tabular}




$\begin{array}{lll}\text { MACVS Religion, M (SD) } & 4.65 & (0.40) \\ \text { MACVS Respect, M (SD) } & 4.24 & (0.49) \\ & & \\ \text { Biopsychosocial beliefs, M (SD) } & .69 & (0.20) \\ \text { Physical causes, M (SD) } & .48 & (0.30) \\ \text { Personality, M (SD) } & .85 & (0.24) \\ \text { Relational issues, M (SD) } & .81 & (0.31) \\ \text { Familial issues, M (SD) } & .70 & (0.42) \\ \text { Trauma, M (SD) } & .82 & (0.29) \\ & & \\ \text { Sociological/spiritual beliefs, M (SD) } & .32 & (0.22) \\ \text { Friends, M (SD) } & .69 & (0.39) \\ \text { American culture, M (SD) } & .36 & (0.42) \\ \text { Prejudice, M (SD) } & .59 & (0.50) \\ \text { Economics, M (SD) } & .48 & (0.43) \\ \text { Spiritual, M (SD) } & .13 & (0.21) \\ \text { Nature disharmony, M (SD) } & .14 & (0.27)\end{array}$

*SES was computed using the Hollingshead Four Factor Index of Social Status (Hollingshead, 1975).

Initial correlation analyses were conducted to examine the relationship between SES and generational status with the independent variables of Anglo orientation (AOS), Latino orientation (LOS), familismo, respeto, spirituality, and traditional gender roles and the primary dependent variables of biopsychosocial beliefs and sociological/spiritual beliefs. The latter were used to determine the presence of any possible covariates that should be controlled for in the exploratory regression analyses (see Table 3).

Generational status was positively correlated with AOS $(r=.53, p \leq .01)$, indicating that participants who are of higher generational status reported more orientation to Anglo culture. Generational status was not associated with any other independent variable or with either of the dependent variables. SES was significantly related to several independent variables. Positive correlations for SES and AOS were found $(r=.58, p \leq$ .01 ), indicating that those with higher SES had more Anglo orientation. Additionally, SES was negatively related to the cultural values of familismo $(r=-.40, p \leq .01)$ and 
traditional gender roles $(r=-.32, p=.01)$, indicating that individuals with higher SES endorsed fewer familismo and traditional gender roles values. SES also was significantly related to the dependent variable sociological/spiritual beliefs $(r=-.33, p=.01)$, indicating that individuals of lower SES reported more sociological/spiritual beliefs about the etiology of ADHD.

Table 3

Correlations for SES and generational status with ARMSA Subscales, MACVS Subscales, and Biopsychosocial and Sociological/Spiritual Beliefs

Generational Status

ARSMA LOS

MACVS Familism

MACVS Gender Roles

MACVS Religion

MACVS Respect

Biopsychosocial beliefs

Sociological/Spiritual beliefs

${ }^{*} p \leq .05,{ }^{* *} p \leq .01,{ }^{* * *} p \leq .001$

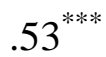

$-.03$

SES

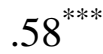

$-.10$

$-.15$

$-.15$

$-.40^{* *}$

$-.32^{*}$

.00

$-.16$

.04

$-.21$

$-.06$

$-.25$

$-.19$

\section{Primary Analyses}

To test our first hypothesis that higher Anglo orientation would be associated with endorsement of more biopsychosocial beliefs, whereas higher Latino orientation would be associated with more sociological/spiritual beliefs about the etiology of ADHD, correlation analyses were conducted (see Table 4). Anglo orientation was not significantly associated with biopsychosocial beliefs $(r=.06, p=.65)$. However, it 
trended toward significance negatively with sociological/spiritual beliefs $(r=-.21, p=$ $.08)$, indicating that participants who reported higher Anglo orientation tended to endorse fewer sociological/spiritual beliefs. Latino orientation was not significantly associated with sociological/spiritual beliefs $(r=.09, p=.44)$, nor with biopsychosocial beliefs $(r=$ $.20, p=.87)$.

To test our second hypothesis that that the cultural values of familismo, respeto, spirituality, and traditional gender roles would be positively related to sociological and spiritual etiological beliefs of ADHD and negatively related to beliefs about biopsychosocial causes, a second set of correlation analyses were conducted (see Table 4). None of the cultural values were significantly correlated with biopsychosocial etiological beliefs. However, several cultural values were significantly correlated with sociological/spiritual beliefs. Familismo was positively associated with sociological beliefs $(r=.28, p=.02)$, suggesting that participants who endorsed strong familismo values also endorsed more sociological/spiritual beliefs for the etiology of ADHD. Traditional gender roles also was positively associated with sociological/spiritual beliefs $(r=.46, p \leq .01)$, indicating that participants who endorsed more traditional gender roles also endorsed more sociological/spiritual etiological beliefs.

Table 4

Correlations for ARMSA Subscales and MACVS Subscales with Biopsychosocial and Sociological/Spiritual Beliefs

Biopsychosocial Sociological/Spiritual

ARSMA AOS

ARSMA LOS

MACVS Familism
.06

.02

.07
$-.21^{+}$

.09

$.28^{*}$ 


$\begin{array}{lcc}\text { MACVS Gender Roles } & .14 & .46^{* *} \\ \text { MACVS Religion } & -.02 & .17 \\ \text { MACVS Respect } & .01 & .10\end{array}$

${ }^{+} p \leq .10,{ }^{*} p \leq .05,{ }^{* *} p \leq .01$

\section{Exploratory and Post-Hoc Analyses}

To examine which cultural factors were most predictive of parental etiological beliefs, exploratory and post-hoc hierarchical multiple regression analyses were conducted to examine the ability of the significant and marginally significant independent variables that emerged from the correlation analyses (i.e., Anglo orientation (AOS), familismo, and traditional gender roles) to predict variance in the dependent variable sociological/spiritual beliefs, as well as in several individual belief categories that make up this overall category. As no significant zero-order correlations emerged for the relationship between the independent variables and biopsychosocial beliefs, regressions were not calculated with biopsychosocial beliefs or any of its individual belief categories as dependent variables.

Sociological/spiritual beliefs overall factor. Hierarchical multiple regression was used to examine the ability of cultural values of familismo and traditional gender roles to predict sociological/spiritual etiological beliefs, after controlling for SES in step 1 and Anglo orientation in step 2 (see Table 5). Anglo orientation was entered into step 2 and the cultural values into step 3 to determine if cultural values, a more cognitive measure of acculturation, could account for significant variance above and beyond that accounted for by Anglo orientation, a behavioral measure of acculturation. SES was entered in step one of the regression, which explained $11.2 \%$ of the variance, and was a 
significant overall model $(F(1,56)=7.04, p=.01)$. Acculturation, specifically Anglo orientation, was entered into step 2, which accounted for $11.2 \%$ of the variance. This overall model was significant $(F(2,55)=3.47, p=.04)$. However, the addition of AOS to the model did not cause a significant change in $\mathrm{R}$ squared $(F$ change $(1,55)=.03, p=$ .87). Finally, the cultural values of familismo and traditional gender roles were entered in step 3, which accounted for an additional 19.3\% (R squared change $=.19)$ and was a significant change $(F$ change $(2,53)=7.35, p \leq .01)$. The overall model was significant $(F(4,53)=5.81, p \leq .01)$ and explained $30.5 \%$ of the total variance. Examination of individual measures indicates that only the cultural value of traditional gender roles was statistically significant in the final model (beta $=.44, p \leq .01$ ).

Table 5

Summary of Hierarchical Regression Analyses for Sociological/Spiritual Beliefs

Sociological/Spiritual Beliefs

$\underline{\text { Variable }}$ $\mathrm{R}^{2} \quad \Delta \mathrm{R}^{2}$ $\beta$

Step 1 $.11^{* *} \quad .11^{* *}$

SES $-.33^{* *}$

Step 2

SES

$.11 \quad .00$

AOS $-.32$

Step 3 $.34^{* *} .23^{* *}$

SES $-.21$

AOS

Familism .08

Gender Roles $.44^{* *}$ 
Sociological/spiritual individual belief categories. Given this robust finding when examining the overall category of sociological/spiritural beliefs, post-hoc analyses were then conducted to further examine predictors of individual belief categories that make up this overall category. Specifically, post-hoc analyses examined the relationship of several independent variables, familismo, traditional gender roles, and spirituality, with sociological/spiritual individual etiological belief categories of friends, spirituality, and nature disharmony. Correlations for each independent variable and sociological/spiritual individual etiological category are presented in Table 6. Multiple regression analyses were conducted for individual belief categories that had a significant correlation with two or more independent variables (see Table 7); thus, post-hoc analyses were conducted to examine the amount of variance predicted in belief categories of friends, spiritual, and nature disharmony, as well as which factors were most predictive of these belief categories.

Table 6

Correlations for Independent Variables with Individual Sociological/Spiritual BAC Categories

\begin{tabular}{|c|c|c|c|c|c|c|}
\hline & A & $\mathrm{B}$ & $\mathrm{C}$ & $\mathrm{D}$ & E & $\mathrm{F}$ \\
\hline Gen.Status & $-.29^{*}$ & -.01 & .01 & -.19 & -.14 & -.07 \\
\hline SES & $-.42^{* *}$ & -.07 & -.01 & -.26 & $-.26^{*}$ & $-.28^{*}$ \\
\hline \multicolumn{7}{|l|}{ ARSMA } \\
\hline AOS & $-.28^{*}$ & -.01 & -.04 & -.17 & $-.21^{+}$ & -.04 \\
\hline LOS & -.01 & -.06 & .15 & .19 & .07 & .10 \\
\hline \multicolumn{7}{|l|}{ MACVS } \\
\hline Familism & .14 & .13 & .10 & .18 & $.24^{*}$ & $.35^{* *}$ \\
\hline Gender Roles & $.25^{*}$ & $.27^{*}$ & $.45^{* *}$ & $.40^{* * *}$ & $.29^{*}$ & .14 \\
\hline Religion & -.16 & .11 & .07 & .08 & $.30^{*}$ & $.29^{*}$ \\
\hline
\end{tabular}


$\begin{array}{lllllll}\text { Respect } & .04 & -.07 & .02 & .14 & .13 & .14\end{array}$

${ }^{+} p \leq .10,{ }^{*} p \leq .05,{ }^{* * *} p \leq .01,{ }^{* * *} p \leq .001$

$\mathrm{A}$ - friends, $\mathrm{B}$ - American culture, $\mathrm{C}$ - prejudice, $\mathrm{D}$ - economics, $\mathrm{E}$ - spiritual, $\mathrm{F}$ - nature disharmony

Friends. Initial exploratory correlation analyses indicated that SES, generational status, Anglo orientation, and traditional gender roles were significantly related to the belief category friends. SES, generational status, and Anglo orientation were all negatively correlated with beliefs about friends contributing to ADHD $(r=-.42, p \leq .01 ; r$ $=-.29, p=.02 ; r=-.28, p=.02$, respectively), indicating that parents who reported lower SES, were of a lower generational status, and who reported less Anglo orientation endorsed more beliefs about the child's friends causing ADHD. Traditional gender roles was positively correlated with beliefs about friends contributing to ADHD $(r=.46, p \leq$ .01 ), indicated that parents who adhere to traditional gender roles also believe that the child's friends contributed to his inattentive and hyperactive-impulsive behavior.

Hierarchical multiple regression analysis was used to examine which variables were most predictive of and the amount of variance predicted in etiological beliefs about friends that could by predicted by the cultural value of traditional gender roles, after controlling for the influence of generational status, SES, and Anglo orientation. Generational status and SES were entered into step 1, AOS was entered into step 2, and traditional gender roles was entered into step 3. The final overall model was significant and accounted for $22.2 \%$ of variance in friend beliefs $(F(4,53)=3.77, p=.009)$. Examination of individual measures indicates that none of the variables made significant individual contributions to the model. 
Table 7

Summary of Hierarchical Regression Analyses With Individual Sociological/Spiritual Belief Categories

Dependent Variable Predictors $\mathrm{R}^{2}$ $\beta$

Friends

$\begin{array}{ll}\text { Step } 1 & \\ & \text { Generational status } \\ & \text { SES }\end{array}$

Step 2

SES

Generational status

SES

AOS

Step 3

Generational status

SES

AOS

Gender roles
$.18^{* *}$

$.18^{*}$

$\begin{array}{ll}-.03 & -.21 \\ -.40^{* *} & -2.75\end{array}$

.00

$-.36^{*}$

$-.10$

.00

$-2.25$

.52

$.22^{* *}$

$-.05$

$-.29$

$-.30$

$-.05$

$-1.86$

.21

$-.26$

1.63

Religious

Nature Disharmony

$\begin{array}{ll}\text { Step } 1 & \\ & \text { SES } \\ \text { Step } 2 & \text { SES } \\ & \text { Familism } \\ & \text { Religion } \\ & \text { Gender Roles }\end{array}$

$.07^{*}$

$.31^{* * * *}$

$-.26^{*}$

$-2.02$

$-.15$

$-1.13$

.01

$.24^{+}$

$.39^{* *}$

.05

1.96

3.02

$\begin{array}{ll}\text { Step } 1 & \\ & \text { SES } \\ \text { Step } 2 & \\ & \text { SES } \\ & \text { Familism } \\ & \text { Religion } \\ & \text { Gender Roles }\end{array}$

$.08^{*}$

$-.28^{*}$

$.26^{* *}$

$-.16$

$-1.17$

.17

$.24^{+}$

1.17

.19

1.82

1.40

${ }^{+} p \leq .10,{ }^{*} p \leq .05,{ }^{* *} p \leq .01,{ }^{* * *} p \leq .001$

Spiritual. Additionally, initial correlation analyses revealed that SES and cultural values of familismo, spirituality, and traditional gender roles were significantly related to spiritual etiology beliefs. SES was negative correlated with spiritual beliefs $(r=-.26, p=$ 
.05), indicating that parents of lower SES reported more spiritual beliefs about the etiology of ADHD. Also, the cultural values of familismo, spirituality, and traditional gender roles were all positively correlated with spiritual beliefs $(r=.24, p=.05 ; r=.30$, $p=.01 ; r=.40, p \leq .01$, respectively), indicating that parents who adhere to these cultural values endorsed more beliefs about spiritual causes. Hierarchical multiple regression analysis was used to examine the amount of variance in spiritual etiological beliefs that could be predicted by the cultural values of familismo, spirituality, and traditional gender roles, after controlling for SES. The final overall model accounts for $31.0 \%$ of the variance in spiritual beliefs $(F(4,53)=5.96, p \leq .01) . \mathrm{R}$ square change $=.24$, suggesting that the cultural values of familismo, spirituality, and traditional gender roles accounted for an additional $24 \%$ of the variance, above the influence of SES. Examination of individual measures indicates that traditional gender roles made a significant individual contribution to the model (beta $=.39, p=.01$ ); religion trended toward significance (beta $=.24, p=.06)$. This suggests that traditional gender roles are important for predicting spiritual etiological beliefs as parents who endorsed this cultural value also endorsed more spiritual beliefs.

Nature disharmony. Finally, initial correlation analyses revealed that SES and cultural values of familismo, spirituality, and traditional gender roles were significantly related to etiology beliefs about nature disharmony. SES was negative correlated with nature disharmony beliefs $(r=-.28, p=.04)$, indicating that parents of lower SES reported more nature disharmony beliefs about the etiology of ADHD. Also, the cultural values of familismo, spirituality, and traditional gender roles were all positively correlated with nature disharmony beliefs $(r=.35, p \leq .01 ; r=.29, p=.02 ; r=.29, p=$ 
.01 , respectively), indicating that parents who adhere to these cultural values endorsed more beliefs about nature disharmony causes. Hierarchical multiple regression analysis was used to examine the amount of variance in nature disharmony etiological beliefs that could be predicted by the cultural values of familismo, spirituality, and traditional gender roles, after controlling for SES, as well as to determine the best predictors of nature disharmony beliefs. The final overall model accounted for $25.8 \%$ of the variance in nature disharmony beliefs $(F(4,53)=4.62, p \leq .001) . \mathrm{R}$ square change $=.183$, suggesting that the cultural values of familismo, spirituality, and traditional gender roles accounted for an additional $18 \%$ of the variance in nature disharmony beliefs. Examination of individual measures indicates that none of the variables made significant contributions to the model; spirituality trended toward significance (beta $=.24, p=.08$ ). 


\section{Discussion}

To address the unmet need for services relating to ADHD among Latino families, the goal of the current study was to examine the role of culture in explaining etiological beliefs about ADHD in Latino parents, as etiological beliefs likely determine what type of services are sought. Specifically, the present study examined how acculturation and several cultural values, familismo, respeto, spirituality, and traditional gender roles, are related to etiological beliefs. Results suggested that acculturation, neither Anglo orientation nor Latino orientation, was related to parental beliefs about the etiology of ADHD. However, cultural values of familismo and traditional gender roles were associated with sociological/spiritual beliefs; none of the variables were associated with biopsychosocial beliefs. Additionally, post-hoc analyses revealed that cultural values were associated with several individual belief categories within the sociological/spiritual domain, including beliefs about friends, spirituality, and nature disharmony. Thus, the current study adds to our knowledge about how Latino parents understand inattentive and hyperactive-impulsive child behavior and has important implications for both research and psychological assessment and treatment with Latino parents. The results support the notion that etiological beliefs and cultural factors that may influence these beliefs are important aspects of help-seeking behavior and therefore, should be included in research examining help-seeking and access to mental health services among Latino families (Eiraldi et al., 2006; Yeh et al., 2005). Additionally, results suggest that the incorporation of alternative etiological beliefs about child behavior may be an important factor in culturally appropriate mental health services. 
Although not the primary focus of the current study, the results also add to our knowledge of an understudied population, that is, Spanish-speaking Latino families living in the United States. Most parents who participated in our study were of Mexican descent, were first generation immigrants, and have been living in the US for more than ten years. Additionally, these parents tended to be of lower SES. They reported strong affiliation toward behavioral aspects of Latino culture, such as listening to Spanish music and eating Latino food, as well as strong affiliation toward Latino cultural values of familismo, respeto, and spirituality and moderate affiliation toward the cultural value of traditional gender roles, as well as toward behavioral aspects of American culture. Finally, they endorsed a variety of etiological beliefs from both the biopsychosocial and sociological/spiritual domains. Parents endorsed more than half of the items within the categories of personality, relational issues, familial issues, and trauma within the biopsychosocial domain, as well as within the categories of friends and prejudice within the sociological/spiritual domain.

\section{Acculturation and Etiological Beliefs}

Contrary to our first prediction, acculturation, neither orientation toward Anglo culture or toward Latino culture, was associated with parental etiological beliefs about ADHD. The measure of acculturation used, the ARSMA-II, focuses on behavioral components of acculturation (e.g., eating American or Latino food, watching TV in English or Spanish, etc.), which may not be as important in understanding parental beliefs as more cognitive measures of acculturation. The ARSMA-II has been used most often in studies of health behavior and has been associated with variables, such as physical activity and problem-solving in a diabetes intervention (Barrera, Toobert, Strycker, \& 
Osuna, 2011) and the rate of unplanned pregnancy among Latina women (Martin \& Garcia, 2011). Thus, it is possible that it may be a more appropriate measure in predicting behavioral outcomes, rather than more cognitive outcomes, such as etiological beliefs as in the current study.

\section{Cultural Values and Etiological Beliefs}

Biopsychosocial beliefs. Our second prediction was partially supported. Contrary to our prediction that cultural values of familismo, respeto, spirituality, and traditional gender roles would be negatively related to endorsement of biopsychosocial causes, none of the variables examined in the current study were significantly associated with this factor of beliefs. Interestingly, on average, parents endorsed about $69 \%$ of the causes in the biopsychosocial categories, which is consistent with research that has shown these beliefs are the most frequently endorsed by all ethnic groups (Yeh et al., 2004), yet cultural values examined in the current study were not associated with these beliefs. Given that the mainstream etiological view of ADHD in the US falls within the biopsychosocial category, reflected in both the current scientific conceptualization its etiology (for a review, see Brassett-Harknett \& Butler, 2007) and Caucasian parental reports of etiological beliefs (Bussing et al., 1998a; Bussing et al., 1998b), Latino parents' endorsement of biopsychosocial beliefs may be reflective of their knowledge and acceptance of these mainstream beliefs. The fact that the cultural values examined in the current study were not associated with endorsement of these views may suggest that biopsychosocial beliefs are not culturally driven for Latino parents.

Sociological/spiritual beliefs. Our prediction that the cultural values of familismo, respeto, spirituality, and traditional gender roles would have a positive 
relationship with sociological/spiritual causes of ADHD was partially supported. Both familismo and traditional gender roles were significantly positively associated with more endorsement of sociological/spiritual beliefs about the etiology of ADHD. Additionally, exploratory analyses revealed that the cultural values predicted significant variance in sociological/spiritual beliefs, even after controlling for SES and acculturation. This suggests that individuals who hold strong Latino cultural values are more likely to believe in etiological factors other than biopsychosocial factors. These factors, such as friends, American culture, prejudice, economic problems, spiritual causes, and nature disharmony, may be particularly salient to Latino families and likely reflect their experiences in the US. Although previous research has not examined cultural variables in relation to etiological beliefs, this finding is generally consistent with research that has shown that Latino parents who endorse these type beliefs are less likely to seek mental health services (Yeh et al., 2005). Latino parents who adhere to traditional Latino values may be reluctant to seek services from mental health providers whose cultural values are inconsistent with theirs or who they view as culturally incompetent (Cauce et al, 2002).

Familismo. To further explore this relationship, post hoc analyses were conducted with individual belief categories within the sociological/spiritual domain and revealed an interesting pattern of findings. Familismo was positively related to several categories of sociological/spiritual beliefs about the cause of child behavior. Specifically, familismo predicted parental beliefs that spiritual causes, as well as disharmony with nature contribute to a child's inattentive and hyperactive-impulsive behavior. Given that familismo is a particularly salient cultural value, its relationship to spiritual and nature disharmony etiological beliefs likely reflects a general Latino cultural perspective, 
suggesting that understanding a parent's cultural values as a whole is important for understanding their etiological perspective.

Interestingly, familismo values were not associated with beliefs about the role of family issues in causing the behavior. Given that familismo emphasizes interdependence and cooperation among family members, it may be unlikely that the family would be viewed as contributing to the problem but rather as a source of support and refuge for the parents (Marín \& Marín, 1991). Additionally, some conceptualizations of familismo have suggested that it represents a socialization process within Latino families in which cultural values are transmitted to new generations through the family (Umaña-Taylor, Alfaro, Bámaca, \& Guimond, 2009). Thus, it is possible that familismo is more of a proximal variable and may influence parental etiological beliefs indirectly, rather than directly, as was measured in the current study.

Spirituality. Additionally, another important predictor of sociological/spiritual etiological beliefs was the cultural value of spirituality, which predicted variance in beliefs about spiritual causes and the role of disharmony with nature. These findings suggest that a parent's spirituality and faith affect how the behavior of their children is interpreted. If religious and/or spiritual beliefs and values are salient to parents, they may be more likely to use these views to understand child behavior and therefore, may be more likely to seek services for problem behavior that are consistent with these views, such as from a priest or spiritual leader (Yeh et al., 2005). Thus, learning about families' spiritual beliefs and values is a necessary step for mental health service providers, and in order to provide culturally-appropriate services, these spiritual beliefs and values should be acknowledge and incorporated into treatment. 
Traditional gender roles. Finally, adherence to traditional gender roles emerged as an important factor in several sociological/spiritual etiological beliefs, friends, spirituality, and nature disharmony. Traditional gender roles was positively associated with beliefs that the child's behavior was influenced by the child's friends. In addition, Anglo orientation was negatively associated with beliefs about the child's friends. Given that the child in the video was male, these associations may reflect Latino parents' understanding of cultural expectations for child behavior, which may be particularly true for parents who are less acculturated to Anglo culture (Cuellar \& Paniagua, 2000). For example, Latino parents may perceive American cultural norms for boys' behavior as more accepting of inattentive and/or hyperactive-impulsive behavior, and therefore, the behavior of the child in the video was thought to be caused by the child's friends, who may be viewed as more inattentive and hyperactive/impulsive in general.

Additionally, the relationship between traditional gender roles and spiritual and nature disharmony etiological beliefs also likely reflects a general Latino cultural perspective, again suggesting that understanding a parent's cultural values as a whole is important for understanding their etiological perspective. The fact that traditional gender roles was a particularly strong variable in predicting several individual categories of etiological beliefs may be reflective of the contrast of this particular value as compared to mainstream American values. Aspects of the other Latino cultural values of familism, respect, and religion may be more consistent with American culture, whereas the value of traditional gender roles is likely inconsistent and in fact may be discouraged in American culture (Cespedes \& Huey, 2008; Santana \& Santana, 2001). Therefore, this value may be particularly important in studies with Latino populations, as it is particularly salient 
and unique to Latino culture. Overall, these results demonstrate the importance of considering parents' cultural values when examining constructs that are likely based on belief systems, such as etiological beliefs of mental health disorders.

\section{Limitations}

Several limitations of the current study should be noted. First, the current study included a relatively homogenous sample of Spanish-speaking, low SES, Mexican parents from Southeast Wisconsin. Future research should replicate these findings in a larger and more geographically representative sample with more variability in Latino subculture. Another limitation of the current study is the limited variability in the measures of Latino values and acculturation (i.e., high means on the majority of the measures), which may have influenced the results. Thus, replicating these finding in a more heterogeneously sample in terms of acculturation is important.

\section{Implications and Future Directions}

Given the gap that exists between the need for services relating to ADHD and service utilization for Latino children, research is needed to address what influences this gap and what can be done to lessen it. One possible way to address the gap is to develop culturally-appropriate assessments and treatments for ADHD. However, as help-seeking models suggest, there are several steps in the process of receiving these services, which include problem recognition, decision to seek help, service selection, and then, service utilization (Eiraldi et al., 2006). Thus, understanding initial stages in this process is essential, as parents need to recognize their child's behavior as problematic, decide to seek help, and choose to seek services from the mental health field, in order for these 
culturally-appropriate services to be utilized. Thus, examination of factors that predict etiological beliefs is an important step in understanding this process, as parental etiological beliefs likely influence the decision to seek help, as well as what services are sought. Results from the current study indicate that traditional Latino cultural values are important in predicting etiological beliefs related to sociological and spiritual causes, as well as in predicting beliefs about the role of friends, spiritual reasons, and disharmony with nature, specifically in causing inattentive and hyperactive-impulsive behavior.

Thus, the current results provide support for the need for more sophisticated examination of ethnic group differences that consider factors that may influence why such differences occur. For example, in research with immigrant populations, incorporation of behavioral acculturation as well as cognitive acculturation, including cultural values, is necessary to gain a complete picture of the acculturation process and its influence on other factors. Additionally, the current results suggest that in order to have a complete understanding of help-seeking behavior, etiological beliefs about mental health disorders need to be examined as part of the help-seeking process in diverse populations. Finally, given that adherence to traditional gender roles was a significant predictor for many belief categories, future research should examine potential differences with parental gender and child gender in relation to etiological beliefs.

These results also should be incorporated into clinical psychosocial assessment and treatments for ADHD in order to establish culturally-appropriate mental health care. In order to receive appropriate mental health care, children in need of mental health services for ADHD must first be recognized as having ADHD and appropriately referred. One way for providers of mental health services to reach parents that may be less likely 
to seek psychological services could be to create partnerships with alternative sources of help, such as schools, churches, or traditional healers, from whom parents may be more likely to seek services initially. Such partnerships could allow for more appropriate referrals to culturally competent mental health service providers. Additionally, etiological beliefs would be important to address in the assessment phase to gain an understanding of how parents and children view the problem. Finally, partnerships with alternative sources of help could allow for each provider to share their particular expertise and create an environment in which families feel their cultural beliefs are acknowledged and welcomed.

Additionally, given that most psychosocial interventions for ADHD work from a biopsychosocial perspective, interventions, such as parent training, should acknowledge alternative etiological perspectives. A more open and dynamic process in which clinicians present their perspective on the etiology but leave room for parents to share their perspectives could allow for the inclusion of ethnic minority parents' etiological beliefs and cultural values. Such steps are necessary to provide culturally-appropriate assessment and treatment for ADHD and bridge the gap between service need and service use among Latino families. 


\section{BIBLIOGRAPHY}

American Psychiatric Association: Diagnostic and Statistical Manual of Mental Disorders, Fourth Edition, Text Revision. Washington, DC, American Psychiatric Association, 2000.

Anderson, R. M. (1995). Revisiting the behavioral model and access to medical care: Does it matter? Journal of Health and Social Behavior, 36, 1-10.

Antshel, K. M. (2002). Integrating culture as a means of improving treatment adherence in the Latino population. Psychology, Health, and Medicine, 7, 435-449. doi: $10.1080 / 1354850021000015258$

Baez, A. \& Hernandez, D. (2001). Complementary spiritual beliefs in the Latino community: The interface with psychotherapy. American Journal of Orthopsychiatry, 71, 408-415.

Bagwell, C. L., Molina, B., Pelham, W. E., \& Hoza, B. (2001). Attention-deficit hyperactivity disorder and problems in peer relationships: Predictions from childhood to adolescence. Journal of the American Academy of Child and Adolescent Psychiatry, 40, 1285-1292.

Barkley, R. A. (2006). Etiologies. In Barkley, R. (Ed.), Attention-Deficit Hyperactivity Disorder (pp. 122-183). New York, NY: Guilford Press.

Barrera, M., Jr., Toobert, D., Strycker, L., \& Osuna, D. (2011). Effects of acculturation on a culturally adapted diabetes intervention for Latinas. Health Psychology. Advance online publication. doi: 10.1037/a0025205

Berry, J. W. (2001). A psychology of immigration. Journal of Social Issues, 57, 615-631

Biederman, J., \& Faraone, S. V. (2006). The effects of attention-deficit/hyperactivity disorder on employment and household income. Medscape General Medicine, 8, 16.

Brassett-Harknett, A. \& Butler, N. (2007). Attention-deficit/hyperactivity disorder: An overview of the etiology and a review of the literature relating to the correlates and lifecourse outcomes for men and women. Clinical Psychology Review, 27, 188-210. doi: 10.1016/j.cpr.2005.06.001

Bussing, R., Gary, F. A., Mills, T. L., \& Garvan, C. W. (2008). Cultural variations in parental health beliefs, knowledge, and information sources related to attentiondeficit/hyperactivity disorder. Journal of Family Issues, 28, 291-318. doi: 10.1177/0192513X06296117. 
Bussing, R., Schoenberg, N. E., \& Perwein, A. R. (1998a). Knowledge and information about ADHD: Evidence of cultural differences among African-American and White parents. Social Science in Medicine, 46, 919-928.

Bussing, R., Schoenberg, N. E., Rogers, K. M., Zima, B. T., \& Angus, S. (1998b). Explanatory models of ADHD: Do they differ by ethnicity, child gender, or treatment status? Journal of Emotional and Behavioral Disorders, 6, 233-242.

Bussing, R., Zima, B. T., Gary, F. A., \& Garvan, C. W. (2003). Barriers to detection, help-seeking, and service use for children with ADHD symptoms. Journal of Behavioral Health Services and Research, 30, 176-189.

Campesino, M., \& Schwartz, G. E. (2006). Spirituality among Latinas/os: Implications of culture in conceptualization and measurement. Advances in Nursing Science, 29, 69-81.

Cauce, A. M., Domenech-Rodriguez, M., Paradise, M., Cochran, B., Shea, J. M., Srebnick, D., Baydar, N. (2002). Cultural and contextual influences in mental health help-seeking: A focus on ethnic minority youth. Journal of Consulting and Clinical Psychology, 70, 44-55. doi: 10.1037//0022-006X.70.1.44.

Cervantes, J. M. \& Parham, T. A. (2005). Toward a meaningful spirituality for people of color: Lessons for the counseling practitioner. Cultural Diversity and Ethnic Minority Psychology, 11, 69-81.

Céspedes, Y.M., \& Huey, S.J. (2008). Depression in Latino adolescents: A cultural discrepancy persepective. Journal of Cultural Diversity and Ethnic Minority Psychology, 14, 168-172. doi: 10.1037/1099-9809.14.2.168

Cuellar, I., Arnold, B., \& Maldonado, R. (1995). Acculturation rating scale for MexicanAmericans-II: a revision of the original ARSMA scale. Hispanic Journal of Behavioral Science, 17, 275-304. doi: 10.1177/07399863950173001

Cuellar, I. \& Paniagua, F. A. (2000). Handbook of Multicultural Mental Health: Assessment and Treatment of Diverse Populations. Academic Press: San Diego, CA.

Drench, M. E., Noonan, A. C., Sharby, N., \& Ventura, S. H. (2007). Psychosocial aspects of health care. Upper Saddle River, New Jersey: Person Prentice Hall.

Derose, K. P. \& Baker, D. W. (2000). Limited English proficiency and Latinos' use of physician services. Medical Care Research and Review, 57, 76-91. doi: $10.1177 / 107755870005700105$

Eiraldi, R. B., Mazzuca, L. B., Clarke, A. T., \& Power, T. J. (2006). Service utilization among ethnic minority children with ADHD: A model of help-seeking behavior. 
Administration and Policy in Mental Health and Mental Health Services Research, 33, 607-622. doi: 10.1007/s10488-006-0076-9

Gidwani, P. P., Opitz, G. M., \& Perrin, J. M. (2006). Mothers' views on hyperactivity: A cross-cultural perspective. Journal of Developmental and Behavioral Pediatrics, 27, 121-126.

Hollingshead, A. A. (1975). Four-factor index of social status. Unpublished manuscript, Yale University.

Johnston, C., \& Mash, E. J. (2001). Families of children with attentiondeficit/hyperactivity disorder: Review and recommendations for future research. Clinical Child and Family Psychology Review, 4, 183-207.

Jorm, A. F. (2000). Mental health literacy: Public knowledge and beliefs about mental disorders. British Journal of Psychiatry, 177, 396-410.

Kataoka, S. H., Zhang, L., \& Wells, K. B. (2002). Unmet need for mental health care among U.S. children: Variation by ethnicity and insurance status. The American Journal of Psychiatry, 159, 1548-1555.

Knight, G. P. et al. (2009). The Mexican American cultural values scale for adults and adolescents. Journal of Early Adolescence. Advance online publication. Doi: $10.1177 / 0272431609338178$

Koneru, V. K., Weisman de Mamani, A. G., Flynn, P. M., \& Betancourt, H. (2007). Acculturation and mental health: Current findings and recommendations for future research. Applied and Preventative Psychology, 12, 76-96.

Lara, M., Gamboa, C., Kahramanian, M. I., Morales, L. S., \& Bautsita, D. E. (2005). Acculturation and Latino health in the United States: a review of the literature and its sociopolitical context. Annual Review of Public Health, 26, 367-397.

LeFever, G. B., Dawson, K. V., \& Morrow, A. L. The extent of drug therapy for attention deficit hyperactivity disorder among children in public schools. American Journal of Public Health, 89, 1359-1364.

Livingston, R. (1999). Cultural issues in diagnosis and treatment of ADHD. Journal of the American Academy of Child and Adolescent Psychiatry, 38, 1591-1594.

Marín G. (1992). Issues in the measurement of acculturation among Hispanics. In Geisinger, K. F. (Ed.), Psychological Testing of Hispanics (pp. 23-51). Washington, DC: American Psychological Association.

Marín, G. \& Gamba, R. J. (2003). Acculturation and changes in cultural values. In Chun, K., Organista, P. \& Marín, G (Eds.), Acculturation: Advances in Theory, 
Measurement, and Applied Research (pp. 83-93). Washington, DC: American Psychological Association.

Marín, G., \& Marín, B. V. (1991). Hispanics: Who are they? In Research with Hispanic Populations (pp. 1-11). Newbury Park, CA: Sage Publications.

Martin, K. R., \& Garcia, L. (2011). Unintended pregnancy and intimate partner violence before and during pregnancy among Latina women in Los Angeles, California. Journal of Interpersonal Violence, 26, 1157-1175. doi: 10.1177/0886260510368154

Marotta, S. A. \& Garcia, J. G. (2003). Latinos in the United States in 2000. Hispanic Journal of Behavioral Sciences, 25, 13-34. doi: 10.1177/0739986303251693

Mendelson, C. (2002). Health perceptions of Mexican American women. Journal of Transcultural Nursing, 13, 210-217.

McLeod, J. D., Fettes, D. I., Jensen, P. S., Pescosolido, B. A., \& Martin, J. K. (2007) Public knowledge, beliefs, and treatment preferences concerning AttentionDeficit/Hyperactivity Disorder. Psychiatric Services, 58, 626-631.

Musgrave, C. F., Allen, C. E., \& Allen, G. J. (2002). Spirituality and health for women of color. American Journal of Public Health, 92, 557-560.

Pham, A. V., Carlson, J. S., Kosciulek, J. F. (2009). Ethnic differences in parental beliefs of Attention-Deficit/Hyperactivity Disorder and treatment. Journal of Attention Disorders. Advance online publication. Doi: 10.1177/1087054709332391

Pelham, W., Fabiano, G., \& Massetti, G. (2005). Evidence-based assessment of attention deficit hyperactivity disorder in children and adolescents. Journal of Clinical Child and Adolescent Psychology, 34, 449-476.

Ramos-Sanchez, L. \& Atkinson, D. R. (2009). The relationship between Mexican American acculturation, cultural values, gender, and help-seeking intentions. Journal of Counseling and Development, 87, 62-71.

Reichman, J. S. (2006). Immigration, acculturation, and health: The Mexican Diaspora. New York, NY: LFB Scholarly Publishing.

Santana, S., \& Santana, F.O. (2001). Mexican culture and disability: Information for US service providers. J. Stone (Ed.). Buffalo, NY: Center for International Rehabilitation Research Information and Exchange.

Schwartz, S. J. (2007). The applicability of familism to diverse ethnic groups: A preliminary study. The Journal of Social Psychology, 147, 101-118. 
Steidel, A. L., \& Contreras, J. M. (2003). A new familism scale for use with Latino populations. Hispanic Journal of Behavior Sciences, 25, 312-330.

Snowden, L. R. \& Yamada, A. (2005). Cultural differences in access to care. Annual Reviews of Clinical Psychology, 1, 143-166.

Srebnik, D., Cauce, A. M., \& Baydar, N. (1996). Help-seeking pathways for children and adolescents. Journal of Emotional and Behavioral Disorders, 4, 210-220.

Umaña-Taylor, A. J., Alfaro, E. C., Bámaca, M. Y., \& Guimond, A. B. (2009). The central role of familial ethnic socialization in Latino adolescents' cultural orientation. Journal of Marriage and Family, 71, 46-60. Doi: 10.1111/j.17413737.2008.00579.x

Yeh, M., Hough, R. L., McCabe, K., Lau, A., \& Garland, A. (2004). Parental beliefs about the causes of child problems: Exploring racial/ethnic patterns. Journal of the American Academy of Child and Adolescent Psychiatry, 43, 605-612.

Yeh, M. McCabe, K. Hough, R. L., Lau, A., Fakhry, F., \& Garland, A. (2005). Why bother with beliefs? Examining relationships between race/ethnicity, parental beliefs about causes of child problems, and mental health service use. Journal of Consulting and Clinical Psychology, 73, 800-807.

Zimmerman, F. J. (2005). Social and economic determinants of disparities in professional help-seeking for child mental health problems: Evidence from a national sample. Health Services Research, 40, 1514-1533. doi: 10.1111/j.1475-6773.2005.00411.x. 